\title{
Management of direct antiviral agent failures
}

\author{
María Buti ${ }^{1,2}$, and Rafael Esteban ${ }^{1,2}$ \\ 'Liver Unit, Department of Internal Medicine, Hospital Universitari Vall d'Hebron and Universitat Autònoma de Barcelona, Barcelona; \\ ${ }^{2}$ Centro de Investigación Biomédica en Red de Enfermedades Hepáticas y Digestivas, Instituto de Salud Carlos III, Madrid, Spain
}

The current standard of care for patients with chronic hepatitis $\mathrm{C}$ virus $(\mathrm{HCV})$ infection is a combination of direct-acting antiviral agents (DAAs). Most HCV patients treated with these drugs achieve viral elimination, but $1 \%$ to $15 \%$ fail to attain this objective. Treatment failures are usually related to relapse, and less often to on-treatment viral breakthrough. $\mathrm{HCV}$ drug resistant associated substitutions are detected in most patients who do not eliminate the virus. The risk of developing these variants depends on host- and virus-related factors, the properties of the drugs used, and the treatment strategies applied. Patients who carry Resistant Associated Substitutions (RASs) may not obtain benefits from treatment, and are at a risk of disease progression. Whether HCV RASs persist depends on their type: NS3-4A variants often disappear gradually after DAA therapy is stopped, whereas NS5A variants tend to persist for more than 2 years. The best way to prevent emergence of resistant variants is to eliminate the virus at the first treatment using highly potent DAAs with genetic barriers to resistance. For those who fail an NS5A inhibitor, deferral of treatment is recommended pending the availability of additional data if they do not have cirrhosis or reasons for urgent re-treatment. If re-treatment is needed, the most commonly used strategy is sofosbuvir as backbone therapy plus a drug from a class other than that previously used, for 24 weeks. Unless it is contraindicated, weight-based ribavirin should also be added. If available, nucleotide-based (eg, sofosbuvir) triple or quadruple DAA regimens may be considered. The optimal treatment for patients who fail an NS5A inhibitor and those with multidrug-resistant variants remains to be defined, and research efforts should continue to focus on treatment for these patients. (Clin Mol Hepatol 2016;22:432-438)

Keywords: Hepatitis C virus; Direct-acting antivirals; Resistant associated substitutions

\section{INTRODUCTION}

Direct-acting antivirals (DAAs) are currently the treatment of choice for chronic hepatitis C virus (HCV) infection.' Several treatment options are available for chronic HCV such as nucleoside polymerase inhibitors like sofosbuvir (SOF), non-nucleoside polymerase inhibitors such as dasabuvir (DSV), protease inhibitors (PIs) such as simeprevir (SMV), paritaprevir boosted with ritonavir (PTVr), and grazoprevir (GZR), and NS5A inhibitors such as ledi- pasvir (LDV), ombitasvir (OBV), daclatasvir (DCV), elbasvir (EBV), and velpatasvir (VEL) have proven highly effective for eradicating $\mathrm{HCV}^{1-3}$ Another $\mathrm{PI}$, asunaprevir (ASV), has been approved in some Asian countries. ${ }^{3}$ These drugs are usually given in combination to increase their effectiveness and prevent the emergence of harbored HCV resistance-associated substitutions (RASs). ${ }^{2}$ The following DAA combination regimens are currently used in clinical practice for patients infected with HCV genotypes (GT) 1 and 4: 1) SMV plus SOF \pm ribavirin (RBV); 2) SOF plus an NS5A inhibitor

\footnotetext{
Abbreviations:

ASV, asunaprevir; BOC, boceprevir; DAA, direct acting antiviral; DCV, daclatasvir; DSV, dasabuvir; EBV, elbasvir; EMA, European Medicines Agency; FDA, Food and Drug Administration; GT, genotype; GZV, grazoprevir; HCV, hepatitis C virus; LDV, ledipasvir; OBV, ombitasvir; P, pegylated interferon; PI, protease inhibitor; PTV/r, paritaprevir boosted with ritonavir; RAV, resistance-associated variant; RASs, resistance-associated substitutions; RBV, ribavirin; SMV, simeprevir; SOF, sofosbuvir; SVR, sustained virologic response; TPV, telaprevir; U.S., United States; RASs, Resistant Associated Substitutions
}

\section{Corresponding author : María Buti}

Liver Unit, Department of Internal Medicine, Hospital Universitari Vall d'Hebron, Passeig Vall Hebron, 119-129, 08035, Barcelona, Spain

Tel: +34-932746559, Fax: +34-934274495

E-mail: mbuti@vhebron.net 
such as DCV or LDV or VEL (the two latter in fixed-dose combinations; 3) an NS3-4A protease inhibitor (PI), paritaprevir boosted with ritonavir (PTV/r) plus an NS5A inhibitor, OBV, in a fixed-dose combination with a non-nucleoside polymerase inhibitor, DSV (in GT4, DSV is not included in the regimen); 4) GZV/EBV in a fixeddose combination; and 5) DCV and ASV in some Asian countries. In patients infected by GT2 and GT3, the only approved regimens are SOF and RBV, and SOF with an NS5 inhibitor either DCV or VEL. In GT5 and GT6, the approved drugs are SOF combined with an NS5A inhibitor. Whichever of these regimens is used, sustained virologic response (SVR) rates in the registration studies involving GT1 and GT4 patients are higher than 90\%, but SVR is lower in GT3 treatment-experienced patients. The safety profiles of these combinations, particularly RBV-free ones are excellent.

The results of treatment with these DAA regimens in various real-world cohorts show high SVR rates (80\%-90\%). Variations in the SVR depend on the number of patients with decompensated liver disease and cirrhosis included in the studies. ${ }^{3-12}$ For patients with GT2 or GT3 infection, the first approved interferon-free regimen was SOF plus RBV for 12 to 24 weeks, which yielded SVR rates of $68 \%$ to $90 \% .^{13-15}$ In addition, three DAA combinations are available for GT3: SOF plus DCV, SOF/LDV and SOF/VEL. ${ }^{3}$ The extensive use of these regimens foresees that a fair number of patients will fail these newer antiviral treatments in the near future.

This review focusses on the management of patients who fail DAA regimens, paying special attention to NS5 failures. The types of failures, the characteristics of patients who fail, the role of HCV drug resistance testing, and the available rescue strategies are reviewed.

\section{VARIABILITY OF HEPATITIS C VIRUS}

Hepatitis $C$ virus has a high turnover rate and an estimated halflife of only 2 to 5 hours: $10^{10}$ to $10^{12}$ virions are produced and cleared per day in an infected patient. ${ }^{16-18}$ Because of the lack of proof-reading activity of the HCV-RNA-dependent RNA polymerase (NS5B) and the high replication activity of $\mathrm{HCV}$, a large number of viral variants are continuously produced during infection (error rate, approximately $10^{-3}$ to $10^{-4}$ mutations per nucleotide per genomic replication). ${ }^{18}$ Most of these variants are cleared by the host's immune system or are unable to replicate because of a functional loss in the encoded proteins, ${ }^{19,20}$ but a large number survive and maintain a chronic infection. The frequency of HCV isolates depends on their replication efficacy and other known and unknown viral and host factors. ${ }^{21}$ The complex mixture of different but closely related genomes comprising the HCV population is known as a quasispecies. Typically, a dominant strain (wildtype) is detectable within the viral quasispecies, along with strains present at lower frequencies. Therapy with DAAs that incompletely suppress viral replication enables selection of preexisting strains with reduced susceptibility to the drugs administered, which play a role in treatment failure. The full clinical impact of HCV drug resistance, its implications in retreatment options, and the potential role of baseline resistance are issues lacking complete answers.

\section{PATTERNS OF ANTIVIRAL FAILURE AND EMER- GENGE OF RESISTANCE-ASSOCIATED VARIANTS}

Failures to DAA combinations in pivotal trials are usually related to relapse, which is defined as serum HCV RNA below the level of quantitation at completion of therapy followed by a rebound to pretreatment levels once therapy is discontinued. Very few patients fail by virological breakthrough, defined by increases in previously undetectable HCV RNA, while they are still on antiviral treatment.

In both situations, virological relapse and breakthrough, a common finding is selection of RASs within the viral quasispecies. ${ }^{22}$ Treatment-emergent RASs with specific amino acid substitutions result in decreases in the effectiveness of a particular drug and represent a developing therapeutic challenge for the clinicians managing these patients. Treatment-emergent RASs will persist depending on the DAAs used. RASs selected during treatment with a PI such as simeprevir are transient and disappear in a few months after discontinuing therapy. For example, in a phase-III trial including patients without cirrhosis, $17 \%$ and $3 \%$ of patients experienced a relapse in the groups receiving 8 weeks and 12 weeks of SMV and SOF respectively. ${ }^{23}$ Among patients with compensated cirrhosis treated for 12 weeks without RBV, 16\% did not achieve SVR, including 3 with viral breakthrough and 13 relapsers. Among 14 patients for whom sequencing data were available, 11 had RASs in the NS3 protease at the time of failure. No sofosbuvir RASs were detected. ${ }^{24}$

RASs related to NS5A inhibitors persist over time and have an impact on the choice and efficacy of future treatment. The typical case is the fixed-dose combination of SOF/LDV. In an integrated analysis of various studies using this regimen, virological failure occurred in $2.4 \%$ of 2144 patients. ${ }^{25}$ NS5A RASs were present in 
$74 \%$ of 51 patients at the time of failure. Most patients included had HCV GT1a. RASs were detected at several positions: M28T,

Table 1. Treatment emergent Resistants Associated Substitutions by Drug Classes

\begin{tabular}{ll}
\hline Drug classes & Specific drug RASs \\
\hline NS3-4A Pls & Simeprevir: R155K, D168V, Q80K (at baseline) \\
& Paritaprevir: R155K, D168V, Y56H \\
& Grazoprevir: R155K, D168V \\
NS5A inhibitors & Ledipasvir: M28T, Q30R, L31M, H58D, Y93H \\
& Ombitasvir: M22T, Q30R, L31M, H58D, Y93H \\
& Daclatasvir: M28T, Q30R, H58D, Y93H \\
& Elbasvir: M28T, L31M, H58D, Y93H \\
& Velpatasvir Q30R/L, L31M, Y93H \\
NS5B polymerase & Sofosbuvir (nucleoside inhibitor): S282T \\
inhibitors & Dasabuvir (nonnucleoside inhibitor): C316Y \\
\hline
\end{tabular}

Table 2. HCV therapeutic regimens for genotype 1

\begin{tabular}{l}
\hline Ombitasvir/Paritaprevir/Ritonavir+Dasabuvir \\
\hline Elbasvir/Grazoprevir \\
Ledipasvir+Sofosbuvir \\
Sofosbuvir+Daclatasvir \\
Sofosbuvir/Velpatasvir \\
Sofosbuvir+Simeprevir \\
\hline
\end{tabular}

Q30R, L31M, H58D, and Y93H (Table 1).

In some Asian countries such as Korea and Japan, the combination of ASV plus DCV is widely used for patients with HCV GT1b infection. This combination is not recommended for patients with GT1a or other genotypes, and it has not been approved in Europe or the United States (Table 2). The main reason is the impact that baseline NS5A RASs have on the SVR. In a pooled analysis including 979 patients with GT1b infection treated with ASV and DCV for 24 weeks, baseline RASs at positions L31F//M/V had a considerable effect on SVR rates: $42 \%$ in 43 patients with RASs versus $88 \%$ in 936 patients without RASs at these positions. ${ }^{26}$ RASs in the NS3 protease did not affect SVR. These results indicate that patients with HCV GT1b with preexisting NS5A RASs at the L31 position should not be treated with this regimen. The rate of virological failures to ASV and DCV was $14 \%$ in 437 patients without cirrhosis and $16 \%$ in 206 patients with cirrhosis. A relevant finding of the studies analyzed was that two-thirds of patients experienced on-treatment breakthrough or no virological response at the end of treatment, whereas one-third relapsed. ${ }^{26}$ In a multinational, phase-III, multicohort study with ASV and DCV the most frequent RASs selected at treatment failure in GT1b patients were at position D168 of the NS3 protease and at position Y93 of the NS5A complex which were present in approximately $75 \%$ of patients. ${ }^{27}$

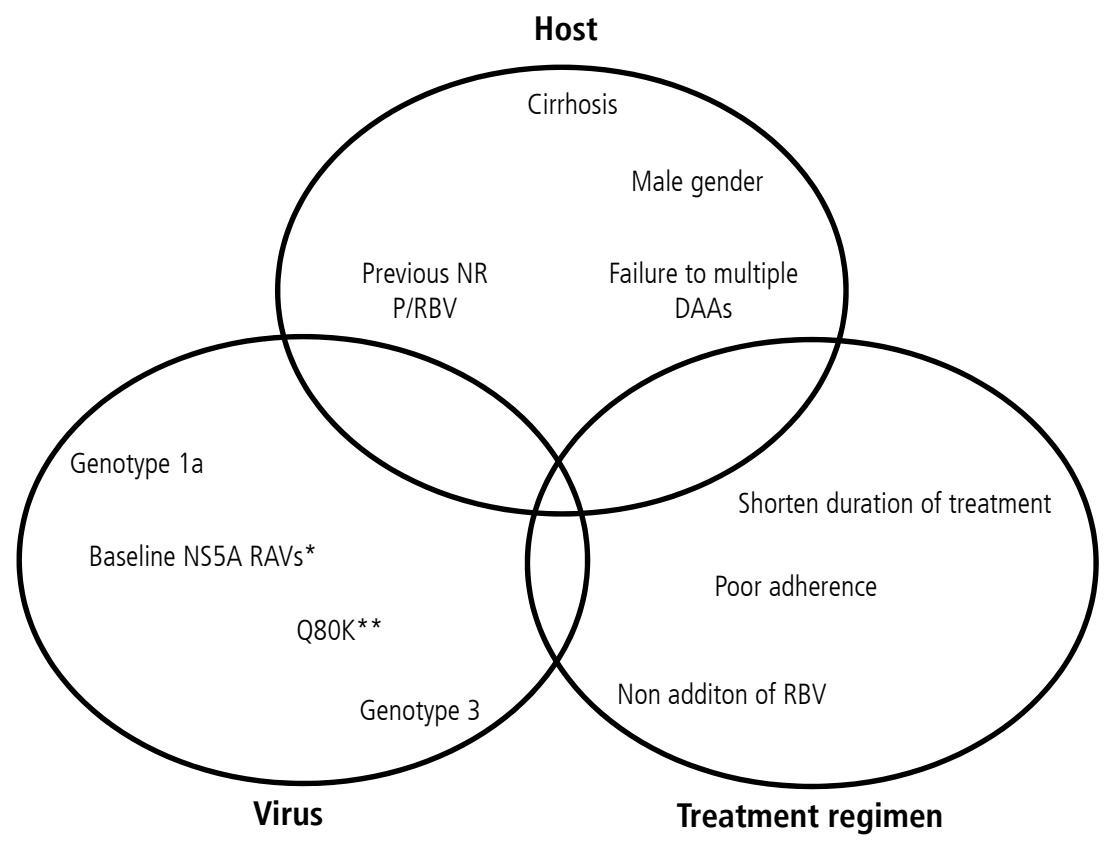

Figure 1. Factors related to the Host, the Virus and Treatment Regimen. 


\section{CAUSES OF TREATMENT FAILURE}

Treatment failure has been related to host factors, viral factors, and treatment-related factors. A combination of two or more factors is needed to result in treatment failure, ${ }^{28}$ as the presence of a single factor has a limited effect on the SVR. Among the host factors, interleukine 28B polymorpyism and cirrhosis are the most imporatntSeveral viral factors have been associated with a low SVR, such as GT1a, GT3, high viral load, and presence of some baseline RASs, but the impact of these factors depends on the DAA regimen used. The treatment-related factors include previous therapy, short therapy duration, and adherence (Fig. 1).

\section{METHODS FOR DETECTING RESISTANCE-AS- SOCIATED VARIANTS}

The genetic diversity of viral populations and resistance-associated variants has been traditionally assessed by population sequencing (ie, direct sequencing of a PCR product) or by clonal sequencing (ie, cloning of a PCR product into plasmid vectors, followed by direct sequencing). The lower limit of detection of direct sequencing ranges from $15 \%$ to $25 \% \cdot{ }^{29}$ Clonal sequencing is more sensitive, but also more expensive and time-consuming. These limitations are overcome by a technique known as deep sequencing, which allows detection of minor variants present at frequencies as low as $0.5 \%{ }^{20}$

At present, none of these techniques are commercially available in Europe, but a number of laboratories are able to perform sequence analysis of the NS3-4A, NS5A, and NS5B regions. In the United States, these tests are commercially available, but they are costly and may not be covered by all insurances companies. Currently, baseline RAS testing to individually tailor therapy is not recommended for treatment-naïve patients. In treatment-experienced patients with some genotypes (eg, GT1a) treated with certain combinations such as GZR and EBR, baseline RAS testing is recommended to decide on therapy duration and whether to include ribavirin in the regimen. ${ }^{3}$ In patients who have failed DAA treatment, testing for resistance-associated variants that confer decreased susceptibility to NS3 protease inhibitors and to NS5A inhibitors is recommended for HCV GT1 patients (regardless of the subtype) who have compensated cirrhosis or a reason for urgent retreatment. ${ }^{3}$ The results of these tests can help in selecting the retreatment drugs and therapy duration.

\section{Retreatment of Failures to Interferon-free DAA regimens}

The number of patients who have failed DAAs is still relatively low, and the available retreatment data do not suffice to establish strong recommendations. The HCV guidance recommendation of the AASLD and IDSA for non-cirrhotic patients who fail an NS5A inhibitor and have no reasons for urgent retreatment is deferral of treatment pending availability of data in HCV GT1 patients. ${ }^{3}$ If retreatment is needed in patients receiving nucleotide-based (eg, sofosbuvir) dual DAA therapy, treatment duration of 24 weeks is recommended, with addition of weightbased ribavirin, unless contraindicated. If nucleotide-based (eg, sofosbuvir) triple or quadruple DAA regimens are available they may also be considered. In this setting, treatment duration ranges from 12 weeks to 24 weeks, and weight-based ribavirin is recommended. Nonetheless, the scientific evidence supporting these recommendations is scarce. The following are the currently available retreatment data for several regimens:

\section{Retreatment of SOF plus SMV failure}

At present, there is relatively little information on salvage therapy for patients failing treatment with SOF plus SMV. However, as cross-resistance between NS3-4A and NS5A RAVs has been ruled out, potential retreatment options are combinations of an NS5A inhibitor plus SOF, such as SOF/LDV or SOF/DCV. ${ }^{30,31}$ The appropriate treatment duration remains to be determined, but 24 weeks will likely be needed in patients with cirrhosis and when RBV is not included. RBV has the potential to shorten the therapeutic regimen to 12 weeks.

\section{Retreatment of SOF plus RBV failure}

Sofosbuvir plus RBV is no longer offered to GT1 patients because the response to this regimen is suboptimal, but it is still a viable option for GT2 and GT3 treatment-naïve patients in some countries. With regard to GT2 and GT3 infection, 107 patients who failed SOF plus RBV in phase-II trials were retreated at the investigator's discretion with SOF plus pegylated interferon (P) and RBV for 12 weeks or SOF plus RBV for 24 weeks. The preliminary results in GT3 patients showed a higher SVR rate in the SOF plus P/RBV 12-week arm (91\% of 25 cases) than in the SOF plus RBV 24-week arm (63\% of 38 cases ). ${ }^{32}$

In the registration studies for SOF/DCV with or without RBV, 13 patients who failed SOF plus RBV have been retreated with SOF/DCV \pm RBV for either 12 or 16 weeks. ${ }^{33,34}$ In clinical prac- 
Table 3. Results of different Re-treatment studies for patients who failed to a NS5A INHIBITOR BASED regimen

\begin{tabular}{llcccc}
\hline Authors & \multicolumn{1}{c}{ Regimen } & $\begin{array}{c}\text { HCV } \\
\text { genotype }\end{array}$ & No cases & SVR12 & $\begin{array}{c}\text { SVR12 in NS5A } \\
\text { RAS cases }\end{array}$ \\
\hline Lawitz, et al. $(2015)^{38}$ & LDV/SOF 24 wks & 1 & 41 & $71 \%$ & $60 \%(18 / 30)$ \\
\hline Hézode, et al. (2016) & SOF+SMV 12 wks & $1 / 4$ & $14 / 2$ & $87 \%$ & $85 \%(11 / 13)$ \\
\hline Poordad, et al. (2016) ${ }^{37}$ & 3D+SOF+RBV 12-24 wks & 1 & 22 & $95 \%$ & $100 \%(17 / 17)$ \\
Gane, et al. (2016) & SOF/VEL+RBV 24 weeks & 1 & 34 & $97 \%$ & $100 \%(6 / 6)$ \\
& & 2 & 14 & $91 \%$ & $100 \%(8 / 8)$ \\
\hline
\end{tabular}

RAS, resistant asocciated sustitutions.

tice, 14 patients were retreated with SOF/DCV \pm RBV or LDV/ SOF and RBV for 24 weeks and all achieved a SVR $12 .^{35} \mathrm{In}$ addition, data on SOF/VEL and RBV for 24 weeks have been reported in 17 GT3 patients. Thirteen of them (77\%) achieved SVR. The presence of NS5A RASs at retreatment had an impact on the SVR. The numbers are small, but SVR was achieved in all patients (3/3) without baseline NS5A RASs compared to $77 \%(10 / 13)$ of those with RASs. ${ }^{36}$

\section{Retreatment of SOF/LDV or SOF/DCV failures}

The SOF/LDV combination is now being widely used. Few patients are expected to fail this therapy, but failure may be an issue in specific populations, such as those with cirrhosis and comorbidities. Patients who have failed SOF/LDV or SOF/DCV harbor NS5A RAVs and may have variants conferring low susceptibility to SOF, although S282T mutations have not been detected in the phase-III studies to date (Table 3).

There is relatively little data on salvage therapy for SOF/LDV failures. Twenty-one patients who failed SOF-based regimens (including SOF/LDV) and harboring NS5B RAVs were retreated with SOF plus P/RBV for 12 weeks. Sixteen (76\%) achieved SVR, a value similar to the retreatment rate in patients without RAVs. $^{35}$ In the 5 patients who relapsed after retreatment, the L159F variant disappeared and V321A was not enriched. ${ }^{35}$

In another study, 41 GT1 patients who failed SOF/LDV, including 19\% with liver cirrhosis, received SOF/LDV for 24 weeks. ${ }^{36}$ Thirty were prior failures to 8 weeks of SOF/LDV and the remaining 11 were failures to 12 weeks of this therapy. Patients previously treated for 8 weeks achieved a higher SVR rate (80\%) than those previously treated for 12 weeks (46\%). Baseline NS5A RAVs, detected in 19 patients, had an impact on SVR rates: SVR was $60 \%$ in the 30 patients with RAVs versus $100 \%$ in the 11 without. Patients having more than two RAVs had a lower SVR rate (50\%) than those with only one
(69\%). The type of single NS5A RAV also influenced SVR, which was lower in patients carrying $\mathrm{Y} 93 \mathrm{H} / \mathrm{N}$ (only 2 of 6 achieved SVR), suggesting that the complexity (number and type) of RAVs present matters. ${ }^{36}$ RBV was not included in the therapeutic regimen, so there was no opportunity to evaluate its potential role in this treatment combination. The above-mentioned study did not include the most difficult to rescue patients, those who fail 24 weeks of SOF/LDV. In these patients, HCV drug resistance testing would be useful for selecting the best salvage therapy, which should include RBV or new, more potent DAAs. The same would be true for failures to SOF/DCV, since the HCV drug resistance should be very similar.

In another study, 22 GT1 patients who failed to respond to DAA therapy, including 16 who were exposed to an NS5A inhibitor, were retreated with SOF and OBV/PTV/r and DSV, with or without ribavirin, depending on the HCV subtype and presence of cirrhosis. The percentages of patients experiencing SVR were $92 \%$ (13/14) after 12 weeks with RBV in GT1a patients without cirrhosis, 100\% (6/6) after 24 weeks with RBV in GT1a patients with cirrhosis, and 100\% (2/2) after 12 weeks without RBV in GT1b patients. ${ }^{37}$

Twenty-three patients with GT1 infection who did not have an SVR after a first course of 4,6 , or 8 weeks of SOF and GZVI EBV were re-treated for 12 weeks with the same combination. All of them achieved SVR. ${ }^{38}$

In another study, 69 patients (37 GT1, 14 GT2, and 18 GT3) who had failed prior VEL-containing regimens in phase-II studies were retreated with SOF/VEL+RBV for 24 weeks. SVR was observed in $97 \%$ of GT1, $91 \%$ of GT2 and $76 \%$ of GT3 patients. The presence of NS5A RASs at retreatment did not have an impact on SVR in GT1 and GT2. No virologic failures occurred in GT3 patients without RASs, but SVR decreased to $77 \%$ in those with NS5A RASs. ${ }^{34}$

Some retreatment data have been reported from clinical 
practice. Fifteen patients who failed to respond to DCV-based regimens were retreated with SOF and SMV \pm RBV for 12 weeks; $87 \%(13 / 15)$ of them achieved SVR, including 8 of 10 with GT1a, 3 of 3 with GT1b, and 2 of 2 with GT4 infection. ${ }^{39}$ In a large European database, 192 DAA failures were identified. Retreatment was started in 23\% ( $n=34)$ and 15\% ( $n=4)$ of GT1 and GT3 patients, respectively. Of these, the majority of GT1 patients had failed SMV/SOF and they were restarted on either OMV, or PTV/r and DSV, or LDV/SOF. In GT3 patients, salvage therapy consisted of 24 weeks of SOF/DCV+RBV. In patients with available outcome data ( $n=14 / 38), 93 \%$ of HCV GT1 and 100\% of GT3-infected patients achieved SVR with salvage therapy. ${ }^{35}$

Two phase-II studies have evaluated SOF/VEL and a new protease inhibitor, voxilaprevir (GS-9857) (100 mg) in a fixeddose combination for 12 weeks in treatment-experienced patients with or without cirrhosis infected with genotypes 1 to 6 , including some who had been previously treated with DAAs. ${ }^{40}$ In total, 128 patients (49\% GT1, 16\% GT2, 27\% GT3, 5\% GT4, and 2\% GT6) were treated. Overall, 27\% were NS5A-experienced, 52\% were non-NS5A DAA-experienced, and 21\% had no prior DAA experience. Baseline RAVs were detected in $60 \%$ of patients ( $20 \%$ NS5A, $15 \%$ NS3, $2 \%$ NS5B (no S 282 T), and $23 \%$ had resistance to multiple classes). All patients except one with GT3 infection achieved SVR. The GT3 patient who relapsed had cirrhosis and the NS5A RAV Y93H, detected at baseline. Relapse occurred at post-treatment week 8 .

In summary, these results obtained from the available published data indicate that current retreatment strategies can lead to SVR in a large percentage of patients who did not achieve SVR with previous DAA regimens, including those with RASs at the time of retreatment. The current evidence indicates that the best retreatment option is the combination of SOF with 1 to 3 other DAAs, as well as addition of ribavirin and/or prolongation of treatment duration to 24 weeks.

\section{Conflicts of Interest}

Maria Buti and Rafael Esteban have received research grants from Gilead and have served as advisors for Gilead, Bristol-Myers Squibb, and Novartis.

\section{REFERENCES}

1. Manns MP, von Hahn T. Novel therapies for hepatitis $C$ - one pill fits all? Nat Rev Drug Discov 2013;12:595-610.

2. Pawlotsky JM. Hepatitis C Virus Resistance to Direct-acting antiviral drugs in interferon-free regimens. Gastroenterology 2016;151:70-86.

3. American association for the study of the liver (AASLD) and Infectious Diseases Society of America (IDSA). HCV Guidance: Recommendations for testing, managing, and treating hepatitis C. AASLD Web site <www.hcvguidelines.org>, Accessed 17-10-2016

4. Jensen DM, O'Leary JG, Pockros PJ, Sherman KE, Kwo PY, Mailliard $M E$, et al. Safety and Efficacy of Sofosbuvir-Containing Regimens for Hepatitis C: Real-World Experience in a Diverse, Longitudinal Observational Cohort. Hepatology 2014;60(Suppl 1):S219.

5. Bacon BR, Dieterich D, Flamm SL, Kowdley KV, Lawitz E, Milligan S, et al. Efficacy of sofosbuvir and simeprevir-based regimens for 304 HCV treatment-experienced patients in a reallife setting; data from the TRIO network. Hepatology 2014;60(Suppl 1):S672.

6. Dieterich D, Bacon B, Flamm S, Kowdley K, Milligan S, Tsai N, et al. Final evaluation of $955 \mathrm{HCV}$ patients treated with 12 week regimens containing sofosbuvir +/- simeprevir in the TRIO network: academic and community treatment of a real-world, heterogeneous population. J Hepatol 2015;62(Suppl 2):S621.

7. Reddy R, Lim JK, Kuo A, Di Bisceglie AM, Vargas HE, Galati JS, et al. All oral HCV therapy is safe and effective in patients with decompensated cirrhosis: interim report from the HCV-TARGET real world experience. J Hepatol 2015;62(Suppl 2):S193.

8. Buggisch P, Sarrazin C, Mauss S, Hinrichsen H, Simon K-G, Vermehren J, et al. Sofosbuvir-based treatment under real life conditions in Germany (The SOFGER trial). J Hepatol 2015;62(Suppl 2):S622.

9. Sulkowski MS, Vargas HE, Di Bisceglie AM, Kuo A, Reddy KR, Lim JK, et al. Safety and Efficacy of Sofosbuvir (SOF) in Combination with Simeprevir (SIM) + Ribavirin (RBV) in Patients with Genotype 1: Interim Results of a Prospective, Observational Study. Hepatology 2014;60(Suppl 1):S660.

10. Pol S, Bourliere M, Lucier S, De Ledinghen V, Zoulim F, DorivalMouly $C$, et al. Safety and efficacy of the combination DaclatasvirSofosbuvir in HCV genotype 1-mono-infected patients from the french observational cohort ANRS CO22 HEPATHER. J Hepatol 2015;62(Suppl 2):S258.

11. Saxena V, Koraishy FM, Sise M, Lim JK, Chung RT, Liapakis A, et al. Safety and efficacy of Sofosbuvir-containing regimens in Hepatitis $C$ infected patients with reduced renal function: real-world experience from HCV-TARGET. J Hepatol 2015;62(Suppl 2):S267.

12. Buggisch P, Petersen J, Wursthorn K, Atanasov P, Gauthier A. Realworld effectiveness of ledipasvir/sofosbuvir 8 weeks chronic hepatitis C treatment. J Hepatol 2015;62(Suppl 2):S280.

13. Lawitz E, Mangia A, Wyles D, Rodriguez-Torres M, Hassanein T, Gordon SC, et al. Sofosbuvir for previously untreated chronic hepatitis $C$ infection. N Engl J Med 2013;368:1878-1887.

14. Jacobson IM, Gordon SC, Kowdley KV, Yoshida EM, Rodriguez-Torres 
M, Sulkowski MS, et al. Sofosbuvir for hepatitis C genotype 2 or 3 in patients without treatment options. N Engl J Med 2013;368:1867-1877.

15. Zeuzem S, Dusheiko GM, Salupere R, Mangia A, Flisiak R, Hyland $\mathrm{RH}$, et al. Sofosbuvir and ribavirin in HCV genotypes 2 and 3. N Engl J Med 2014;370:1993-2001.

16. Lontok E, Harrington P, Howe A, Kieffer T, Lennerstrand J, Lenz O, et al. Hepatitis C Virus Drug Resistance-associated Substitutions: State of the Art Summary. Hepatology 2015;62:1623-1632.

17. Holland J, Spindler K, Horodyski F, Grabau E, Nichol S, VandePol S. Rapid evolution of RNA genomes. Science 1982;215:1577-1585.

18. Martell M, Esteban JI, Quer J, Genescà J, Weiner A, Esteban R, et al. Hepatitis $C$ virus (HCV) circulates as a population of different but closely related genomes: quasispecies nature of HCV genome distribution. J Virol 1992;66:3225-3229.

19. Bartenschlager R, Lohmann V. Replication of hepatitis C virus. J Gen Virol 2000;81:1631-1648.

20. Gregori J, Esteban JI, Cubero M, Garcia-Cehic D, Perales C, Casillas $R$, et al. Ultra-deep pyrosequencing (UDPS) data treatment to study amplicon HCV minor variants. PLoS One 2013;8:e83361.

21. Vermehren J, Sarrazin C. The role of resistance in HCV treatment. Best Pract Res Clin Gastroenterol 2012;26:487-503.

22. Pawlotsky JM. Treatment failure and resistance with direct-acting antiviral drugs against hepatitis C virus. Hepatology 2011;53:17421751.

23. Kwo P, Gitlin N, Nahass R, Bernstein D, Etzkorn K, Rojter $S$, et al. Simeprevir plus sofosbuvir (12 and 8 weeks) in hepatitis $C$ virus genotype 1-infected patients without cirrhosis: OPTIMIST-1, a phase 3, randomized study. Hepatology 2016;64:370-380

24. Lawitz E, Matusow G, DeJesus E, Yoshida EM, Felizarta F, Ghalib R, et al. Simeprevir plus sofosbuvir in patients with chronic hepatitis C virus genotype 1 infection and cirrhosis: A phase 3 study (OPTIMIST2). Hepatology 2016;64:360-369.

25. Sarrazin C, Dvory-Sobol H, Svarovskaia ES3, Doehle BP, Pang PS, Chuang SM, et al. Prevalence of Resistance-Associated Substitutions in HCV NS5A, NS5B, or NS3 and Outcomes of Treatment With Ledipasvir and Sofosbuvir. Gastroenterology 2016;151:501-551

26. Kao JH, Jensen DM, Manns MP, Jacobson I, Kumada H, Toyota J, et al. Daclatasvir plus asunaprevir for HCV genotype $1 \mathrm{~b}$ infection in patients with or without compensated cirrhosis: a pooled analysis. Liver Int 2016;36:954-962.

27. Manns M, Pol S, Jacobson IM, Marcellin P, Gordon SC, Peng CY, et al. All-oral daclatasvir plus asunaprevir for hepatitis $C$ virus genotype 1b: a multinational, phase 3, multicohort study. Lancet 2014:384:1597-1605.

28. Buti M, Riveiro-Barciela M, Esteban R. Management of direct-acting antiviral agent failures. J Hepatol 2015;63:1511-22.

29. Thielen A, Martini N, Thiele B, Däumer M. Validation of HIV-1 drug resistance testing by deep sequencing: insights from comparative
Sanger sequencing. Antivir Ther 2014;19(Suppl 1):A78

30. Bourliere M, Bronowicki JP, de Ledinghen V, Hézode C, Zoulim F, Mathurin $\mathrm{P}$, et al. Ledipasvir-sofosbuvir with or without ribavirin to treat patients with HCV genotype 1 infection and cirrhosis nonresponsive to previous protease-inhibitor therapy: a randomised, double-blind, phase 2 trial (SIRIUS). Lancet Infect Dis 2015;15:397404.

31. Wyles D, Pockros P, Morelli G, Younes Z, Svarovskaia E, Yang JC, et al. Ledipasvir-sofosbuvir plus ribavirin for patients with genotype 1 hepatitis C virus previously treated in clinical trials of sofosbuvir regimens. Hepatology 2015;61:1793-1797.

32. Esteban R, Nyberg L, Lalezari J, Ni L, Doehle B, Kanwar B, et al. Successul retreatment with sofosbuvir-containing regimens for $\mathrm{HCV}$ genotype 2 or 3 infected patients who failed prior Sofosbuvir plus ribavirin therapy. J Hepatol 2014;60(Suppl 1):S4-S5.

33. Nelson DR, Cooper JN, Lalezari JP, Lawitz E, Pockros PJ, Gitlin N, et al. All-oral 12-week treatment with daclatasvir plus sofosbuvir in patients with hepatitis C virus genotype 3 infection: ALLY-3 phase III study. Hepatology 2015;61:1127-1135.

34. L Leroy V, Angus P, Bronowicki JP, Dore GJ, Hézode C, Pianko S, et al. Daclatasvir, sofosbuvir, and ribavirin for hepatitis $C$ virus genotype 3 and advanced liver disease: A randomized phase III study (ALLY-3+). Hepatology 2016;63:1430-1441

35. Vermehren J, Susser S, Dietz J, von Hahn T, Petersen J, Hinrichsen H, et al. Retreatment of patients who failed DAA-combination thrapies: real-world experience from a large hepatitis $C$ resistance database. J Hepatol 2016;64(Suppl 2);S188.

36. Gane E, Shiffman ML, Etzkorn K. Sofosbuvir/velpatasvir in combination with ribavirin for 24 weeks is effective retreatment for patients who prior NS5A containing DAA regimens: results of the GSUS-342-1553 study. J Hepatol 2016; 64(Suppl 2):S147.

37. Poordad F, Bennett M, Sepe TE, Cohen E, Reindollar RW, Everson G, et al. Ombitasvir/paritaprevir/r, dasabuvir, and sofosbuvir treatment of patients with HCV genotype-infection who failed a prior course of DAA therapy: the quartz-I study. J Hepatol 2016;64(Suppl 2):S767.

38. Lawitz E, Poordad F, Gutierrez JA, Wells JT, Landaverde CE, Reiling JR, et al. C-SWIFT Retreatment (Part B): 12 weeks of elbasvir/ grazoprevir with sofosbuvir and ribavirin successfully treated GT1infected subjects who failed short-duration all-oral therapy. J Hepatol 2015;62(Suppl 2):1386A

39. Hézode C, Chevaliez S, Scoazec G, Soulier A, Varaut A, BouvierAlias $M$, et al. Retreatment with sofosbuvir and simeprevir of patients with hepatitis C virus genotype 1 or 4 who previously failed a daclatasvir-containing regimen. Hepatology 2016;63:1809-1816.

40. Gane E, Kowdley KV, Pound D, Stedman CA, Davis M, Etzkorn K, et al. Efficacy of sofosbuvir, velpatasvir, and GS-9857 in patients with HCV genotype 2, 3, 4, or 6 Infections in an open-label, phase 2 trial. Gastroenterology 2016 Jul 30. [Epub ahead of print] 